\title{
RETRACTED ARTICLE: Utilizing a deep learning model to enhance video credibility verification system
}

\author{
Yongjun Zheng ${ }^{1} \cdot$ Yinjie Wei ${ }^{1} \cdot$ Wenjun $\mathrm{Li}^{1} \cdot$ Yi Lu ${ }^{1}$
}

Received: 12 October 2017 /Revised: 19 October 2017 /Accepted: 25 October 2017 /

Published online: 16 November 2017

(C) Springer Science+Business Media, LLC 2017

The Editor-in-Chief has retracted this article [1], which was published as part of special issue "Multi-source Weak Data Management using Big Data", because there is evidence of figure duplication without appropriate permission, as well as evidence suggesting authorship manipulation and an attempt to subvert the peer review process.

Corresponding author Yongjun Zheng stated on behalf of all co-authors that they agree to this retraction.

\section{References}

1. Zheng, Y., Wei, Y., Li, W. et al. Utilizing a deep learning model to enhance video credibility verification system. Multimed Tools Appl (2017). https://doi.org/10.1007 /s11042-017-5360-z

Electronic supplementary material The online version of this article (https://doi.org/10.1007/s11042-017$5360-\mathrm{z}$ ) contains supplementary material, which is available to authorized users.

Yongjun Zheng

zhengyongjun1778@126.com

1 College of Metrology and Measurement Engineering, China Jiliang University, Hanghzhou, Zhejiang, China 